\title{
The Combination of Steganography and Cryptography for Medical Image Applications
}

\author{
B.Kiran Bala, R.Sasi Kumar \\ Department of Computer Science and Engineering, K.Ramakrishnan College of Engineering, Trichy, Tamil Nadu, India
}

\begin{tabular}{l}
\hline \hline Article Info \\
\hline Article history: \\
Received Jan 5, 2018 \\
Revised May 2, 2018 \\
Accepted May 27, 2018 \\
\hline
\end{tabular}

Keyword:

Authentication

Cryptography

Mammogram Image

Steganography
Copyright (C) 2018 Institute of Advanced Engineering and Science. All rights reserved.

\section{Corresponding Author:}

B.Kiran Bala,

Department of Computer Science and Engineering,

K.Ramakrishnan College of Engineering,

Trichy, Tamil Nadu, India.

Email: kiranit2010@gmail.com

\section{INTRODUCTION}

The security is the very big issue in present technology to overcome those issue in various field day by day so many new proposed technique is released every day but in the field of medical the security is very much important to give privacy and security for the patient details to avoid data hacking and data surfing in the hospital database even though hospital staff should not directly see the without permission of patient as well as doctor. The Proposed system deals with the MRI scan image for the patient and it should be preprocessed and then convert into steganography technique it is in the form of two things first one is in image form another type is in form of text form each time MRI image has been converted into text or image form [1-2].

The next step for the proposed system is from that using the proposed cryptography algorithm just convert those unknown form into another form for the purpose of security aspect to give privacy and authentication for the patient as well as hospital for high quality of service. MIAS database is used for the proposed system from that database the entire process has been carried out for the better result for the present technology result. The proposed system is not deals with the diagnosis of the disease from the image but the system is mainly focus on the security aspect of the system[3][4]. Basic diagram for proposed system as shown in Figure 1. 


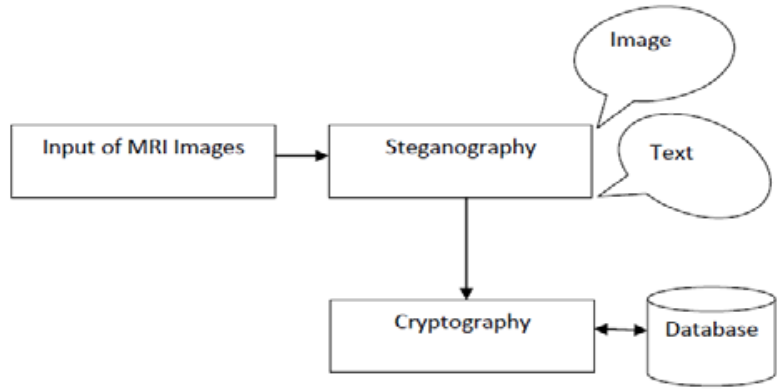

Figure 1. Basic diagram for proposed system

The proposed system is having two modules namely steganography form and cryptography module from the input image random chosen of image or text from the proposed system and then by using image conversion means MRI image has been converted into binary form and then plain image from the proposed algorithm identify the unused space from the plain image insert the MRI image into the plain image and now the MRI image is in unknown form and then encrypt the image into cipher text and store in the database. Likewise, if MRI image can also be convert into text and encrypt into cipher text and finally store into database [5].

\section{METHODOLOGIES AND RESULTS}

\subsection{Initial Process for Image}

The First and foremost process in the proposed system after getting the MRI image the system will random conversion of image or text from of the MRI image[6]. Random conversion for steganography process as shown Figure 2.

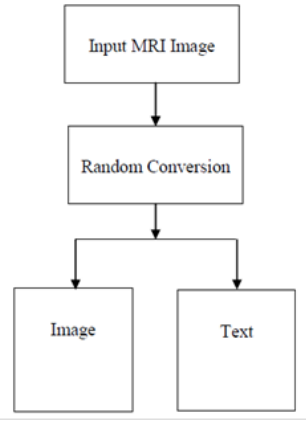

Figure 2. Random conversion for steganography process

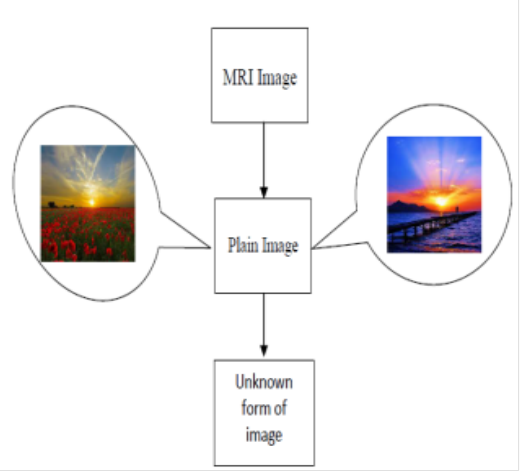

Figure 3 Basic diagram of image for Steganography process

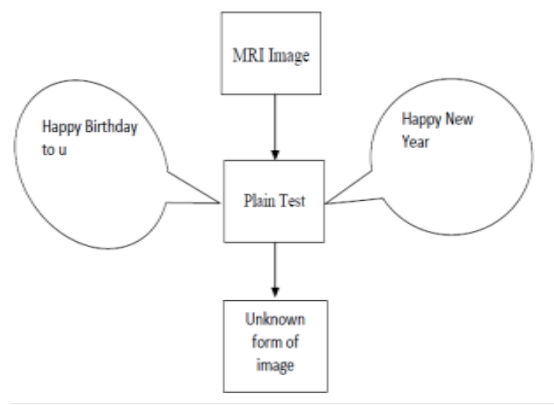

Figure 4. Basic diagram of text for steganography process 
The Figure 3 and Figure 4 for the process of steganography has been shown in the Figures and finally store in the database of the proposed system[7]

\section{RESULTS AND DISCUSSION}

The Proposed system have implementation of the entire process and which has process and execution time accuracy has been discussed in the system. The result of proposed system are available in Table 1

Table 1. Implementation Result of Proposed System

\begin{tabular}{ccc}
\hline Image Type & Time (in sec) & Accuracy (in \%) \\
\hline JPEG & 0.001 & 99.3 \\
MPEG & 0.001 & 99.4 \\
BMP & 0.002 & 99.5 \\
PNG & 0.001 & 99.5 \\
GIF & 0.001 & 99.4 \\
PNM & 0.001 & 99.5 \\
BAT & 0.003 & 99.3 \\
BPG & 0.001 & 99.4 \\
\hline
\end{tabular}

From the implementation part the discussion has been made that the input image converted and result as well as graphs are available in the Figure 5 and Figure 6 for the proposed system.

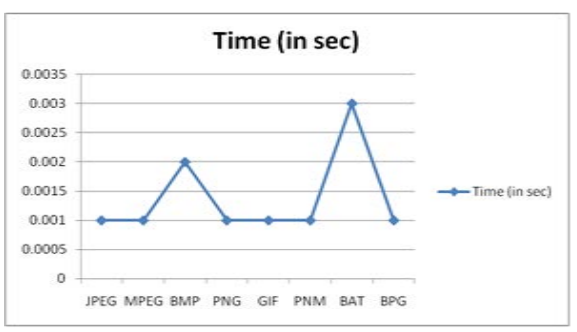

Figure 5. Graph for image type \& time taken for conversion

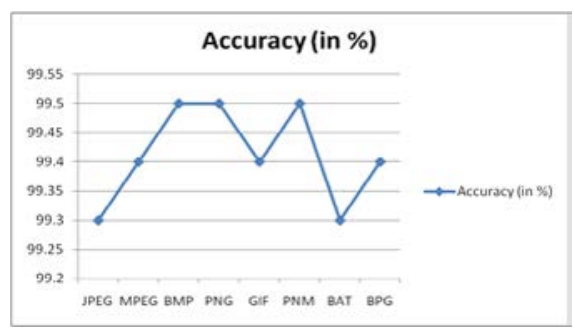

Figure 6. Graph for image type \& accuracy from the conversion

\section{CONCLUSION}

The experimental results reported in this paper shown in the Figure 5 and Figure 6 for the processing and efficiency of the entire proposed system is possible the justification shown in the implementation part is available. The proposed system deals with security of the present technology and which gives the input for the issues in the present system and solution to the issues of the present technology for the biomedical application for the medical sector for privacy and highly confidentially data of the patient.

\section{REFERENCES}

[1] http://www. mammoimage. Org / databases/

[2] B.Kiran Bala, R.Sasikumar, Identification Of Cancer From The Mammogram Images By Using Frequency Domain Approaches, International Journal of ChemTech Research, April 2017, Volume 10 No.5.

[3] B.Kiran Bala, A Novel Approach to Identify the Micro calcification Images, Journal of Chemical and Pharmaceutical Sciences, SpecialIssue2: February 2017, Pages 190-192.

[4] B.Kiran Bala, Biometrics for Mobile Banking, International Journal of Technology And Engineering System, 2011, Volume 2, Issue 1,Pages95-97.

[5] B.Kiran Bala, T.m.nithya, Remedy For Disease Affected Iris In Iris Recognition, International Journal of Research in Engineering and Technology, November Issue 2012, ISSN: 2319 - 1163, page No. 332-334.

[6] B.Kiran Bala, A Novel Approach to Generate a Key for Cryptographic Algorithm, Journal of Chemical and Pharmaceutical Sciences, Special Issue 2: February 2017, Pages 229-231 